\title{
Quality Enhancement in Image Enlargement
}

\author{
Francescomaria Marino, Giuseppe Mastronardi \\ Dipartimento di Elettrotecnica ed Elettronica, Politecnico di Bari \\ Via Re David, 200 - 70125 Bari (Italy)
}

\begin{abstract}
Interpolation methods, usually employed in image enlargement, cause a degradation of the image quality in proximity to the edges. In fact, the best interpolation leads to fuzziness effects often due to the image features ignored during the process. In order to have a contained definition loss after the enlargement operation, it is necessary to consider the original image as a subsampled image of the enlarged one. It follows that the image needs to be represented by its low and high frequency components together. A method able to consider and provide the above mentioned image components is the bidimensional Fast Fourier Transform (2D-FFT). In the paper a strategy of partitioning \& overlapping is proposed to reduce the computational complexity, conditioned by the number and the accuracy of the operations, when the 2D-FFT is applied to the whole image. So, the image enlargement achieves the goal with low definition loss and without expensivecomputational load. The quality enhancement is evaluated in terms of extracted edges.
\end{abstract}

\section{Introduction}

The expansion operators are very often used in the field of image processing, mainly as a preliminary approach to the local spatial processing. Interpolation methods are usually employed for this aim; nevertheless, they cause a degradation of the image quality in proximity to the edges. The interpolation, however applied, leads to fuzziness effects mainly due to the contrast smoothing, since it produces the absent information (for instance: 3 new pixels calculated on 4) starting only from the luminance values of the original image.

In order to avoid the definition loss after the image enlargement, it is necessary to consider the original image as a subsampled image (for instance: 1 pixel on 4) of the enlarged one. It follows that the image needs to be represented by its low and high frequency components together. The low frequency components are obtained by the luminance of the window, selected in the source image. The other components are inserted in order to achieve a better definition by generating an artificial texture.

A method able to provide the above mentioned image components is the bidimensional Fast Fourier Transform (2D-FFT). The enlargement operation by Fourier transform is usually achieved by expanding the image transform with zero values and then appling the inverse transform to the matrix so obtained. During an enlargement $\mathrm{x} 4$ of $N \times \mathrm{x}$ pixel images, this algorithm requires

$$
N^{2} \lg _{2}(N)+4 N^{2} \lg _{2}(2 N)=N^{2}\left(5 \lg _{2}(N)+4\right)
$$

complex multiplications. Moreover, in the case of large value of $N$, the angles in the goniometric circle are similar, so an high precision in the representation of the Fourier coefficients is necessary: consequently, the computational complexity increases. From the hardware point of view, a very large size of memory is required, too [1]. 


\section{The Algorithm}

To spare the high computational complexity due to 2D-FFT, when applied to the whole image, a first strategy could be adopted partitioning the original image in $2 \times 2$ pixel windows and using them as source image within the classic 2D-FFT approach. Nevertheless the set of inverse FFTs generates an enlarged image with an unseemly "mosaic", caused by the boundary effect of the contiguous antitransforms (evaluations about this method and other ones are reported in [2]).

To overcome the above mentioned chequered effect, an overlapping strategy is proposed. This one has been approached in [3] by using the Hadamard transform in the same enlargement operation, with results good about the computational load, but not excellent concerning the image quality.

If we call $P_{r, c}$ the grey level of the pixel belonging to the $r^{\text {th }}$ raw $(r=0, \ldots, M-1)$ and to the $c^{\text {th }}$ column $(c=0, \ldots, N-1)$ of the source image, our algorithm (o.a.) is composed by the phases sketched and explained in the following:

$$
\begin{aligned}
& B_{r, c} \equiv\left[\begin{array}{cc}
P_{r-1, c-1} & P_{r-1, c-1} \\
P_{r, c-1} & P_{r, c}
\end{array}\right] \equiv\left[\begin{array}{cc}
P_{1} & P_{2} \\
P_{4} & P_{3}
\end{array}\right] \stackrel{2 \times 2 F F T}{\rightarrow} T_{r, c} \equiv\left[\begin{array}{cc}
T_{1} & T_{2} \\
T_{4} & T_{3}
\end{array}\right] \rightarrow \\
& \stackrel{R_{i}=K_{R} \cdot T_{i}}{S_{i}=K_{S} \cdot T_{i}} \longrightarrow\left[\begin{array}{cccc}
R_{1} & S_{1} & S_{2} & R_{2} \\
S_{1} & T_{1} & T_{2} & S_{2} \\
S_{4} & T_{4} & T_{3} & S_{3} \\
R_{4} & S_{4} & S_{3} & R_{3}
\end{array}\right] \stackrel{4 \times 4 I F F T}{\longrightarrow}
\end{aligned}
$$

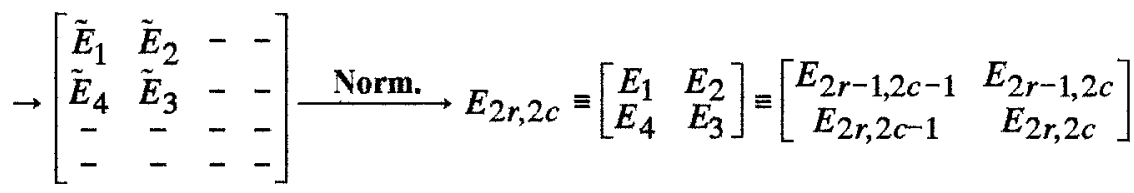

1) to consider the $2 \times 2$ block $B_{r, c}$ for each $P_{r, c}(r=1, \ldots, N-1 ; c=1, \ldots, N-1)$;

2) to compute for each block $B_{r, c}$ its Fast Fourier Transform $T_{r, c}$;

3) to extrapolate, for each term $T_{i}$, the high frequency components $R_{i}$ and $S_{i}$ by the formulas $R_{i}=K_{R} \cdot T_{i}$ and $S_{i}=K_{S} \cdot T_{i}$ using suitable value for $K_{R}$ and $K_{S}$;

4) to compute for each block so obtained the four lowest components of its Inverse Fast Fourier Transform;

5) to normalize these components and adopt them as new pixels for the $E_{2 r, 2 c}$ block;

6) to mosaic these blocks in order to achieve an enlargement $x 4$ of the selected window.

This enlargement $\mathrm{x} 4$ is obtained because for each $P_{r, c}$ pixel in the original image, one block $E_{2 r, 2 c}$ (composed by four pixels) is generated. The enlarged image will be carried out by mosaiking the blocks $E_{2 r, 2 c}$.

Moreover, the propagation error is strongly reduced, because the 2D-FFT required by this approach are computed on $(N-1)^{2}$ windows of $2 \times 2$ (direct transform) and $4 \times 4$ (inverse transform) pixels by adding a little number of terms: in these cases, the Fourier coefficients require a very short precision. 
The number of complex multiplications, required in order to compute $(N-1)^{2} 2 \times 2$ (full) and $4 \times 4$ (partial) 2D-FFT, is

$$
4(N-1)^{2}+8(N-1)^{2}=12(N-1)^{2}
$$

this one is less than that one required for a non partitioned 2D-FFT processing, above all, without high accuracy requiring or large size of memory employing.

At last, the partitioning of the whole image reaches two others fundamental advantages respect to the classic 2D-FFT approach:

a) to implement the algorithm in parallel way;

b) to consider images not needfully having power-of-2 dimensions.

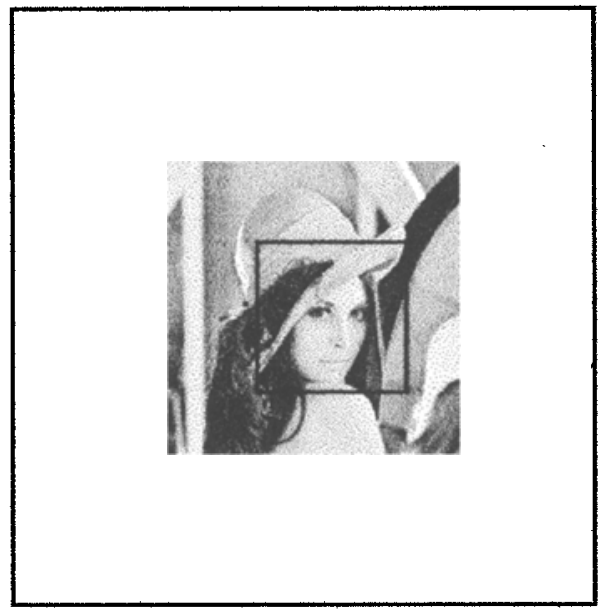

Fig. 1. The portrait of "Lenna" with the selected window to be enlarged.

\section{Conclusions and Remarks}

We have used as source image a $128 \times 128$ pixel window of the famous portrait of "Lenna" shown in Fig. 1. In Fig. 2 some enlargements $x 16$ are shown in order to compare the results of the respective elaborations. They are obtained iterating twice the base process to make more evident the effects of the implemented solutions.

The enlargements in Fig. 2 are respectively obtained: 2 a) by bicubic interpolation; 2b) by o.a. with $K_{R}=K_{S}=1 ; 2$ c) by o.a. with $K_{R}=0.1, K_{S}=\sqrt{2} K_{R} ; 2$ d) by o.a. with $K_{R}=0.05, K_{S}=\sqrt{2} K_{R} ; 2$ e) by o.a. with $K_{R}=0.025, K_{S}=\sqrt{2} K_{R} ; 2$ f) by o.a. with $K_{R}=K_{R}=0$. The ratio $K_{S} / K_{R}$ is obtained in experimental way.

The quality enhancement is evaluated in terms of extracted edges: they are shown in Fig. 3. The algorithm adopted to extract the edges is a spatially adaptive filter reported in [4].

The bicubic law, used in the interpolation for the enlargement in Fig. 2a, produces a very low definition loss [5]; the image, so generated, appears similar to that one shown in Fig. 2f, that is obtained by o.a. with null values of both parameters $K_{R}$ and $K_{S}$ (see Fig. 3a vs. Fig. 3f).

A proper use of the $K$-parameters generates an artificial texture. This effect, proportional to the $K$-parameters, produces a better definition of particulars in no strongly contrasted areas. 
Taking care of the texture does not become preponderant (as in the Figg. $3 \mathrm{~b}$ and 3c), significant results can be obtained. In fact, by a parametric use, it is possible to adapt the enlargement quality to the image features in order to thin the fuzziness effect. For the image under consideration, the Figg. $3 \mathrm{~d}$ and $3 \mathrm{e}$ give prominence that values of $K_{R}=0.025 \div 0.05$ can be suitably used.

\section{References}

1. A. V. Oppenheim, R.W. Schafer: Digital Signal Processing. (Prentice Hall International Inc., New Jersey, 1975).

2. F. Marino, G. Mastronardi: Techniques of Image Enlargement. Invited paper at the $18^{\text {th }}$ Intemational Conference MIPRO'95, sponsored by IEEE (Croatian Section), Rijeka, Croatia, May 22-25, 1995, MIS 2, pp. 1-13.

3. G. Mastronardi, F. Spilotros: Image Expansion Operators based on the Hadamard Pyramidal Coding. Progress in Image Analysis and Processing III, (Edited by S. Impedovo, World Scientific, 1993), pp. 111-114.

4. G. Mastronardi, S. Sangiovanni: An Adaptive Filter for Image Processing in OCCAM2. Proc. of the ISMM Int. Symp. on Industrial, Vehicular and Space Applications of Microcomputers, New York, 1990, pp. 69-72.

5. M. Sonka, V. Hlavac and R. Boyle: Image Processing, Analysis and Machine Vision. (Chapman \& Hall, Cambridge, 1994). 


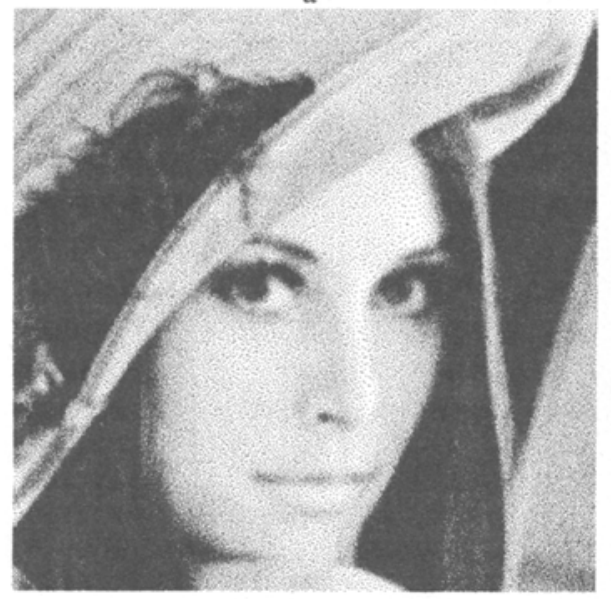

c

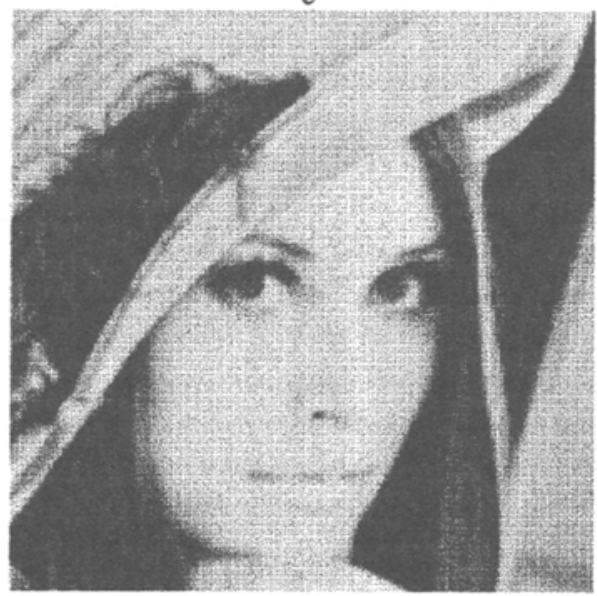

$\mathrm{e}$

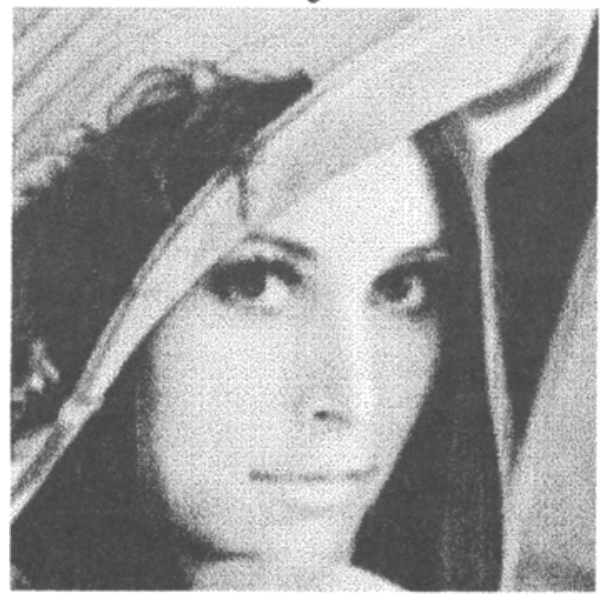

b
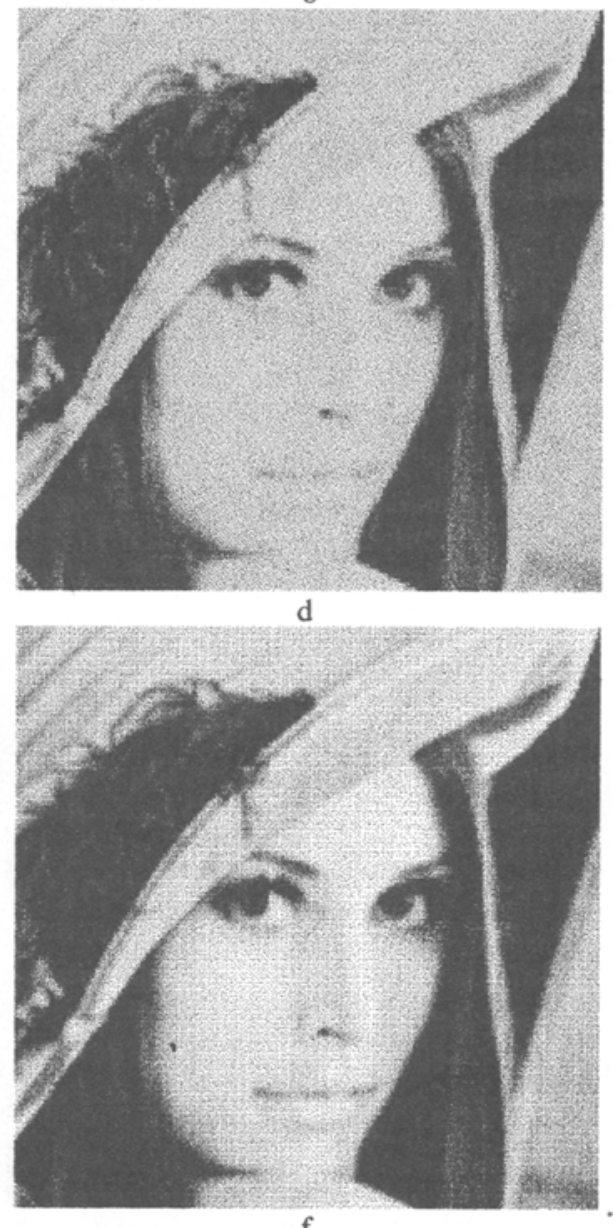

f

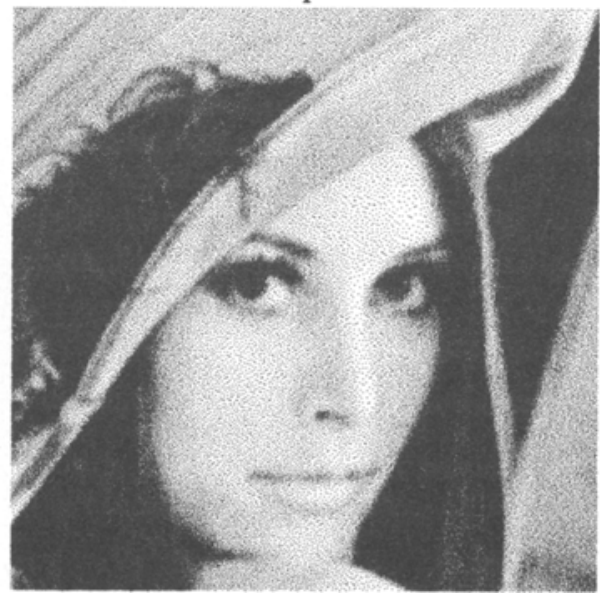

Fig. 2. Enlargements $\times 16$ of $128 \times 128$ pixels of "Lenna": a) by bicubic interpolation; b) by o.a. with $K_{R}=K_{S}=1$; c), d), e) by o.a. respectively with $K_{R}=0.1,0.05,0.025$ and $K_{S}=\sqrt{2} K_{R} ;$ f) by o.a. with $K_{R}=K_{S}=0$. 
a

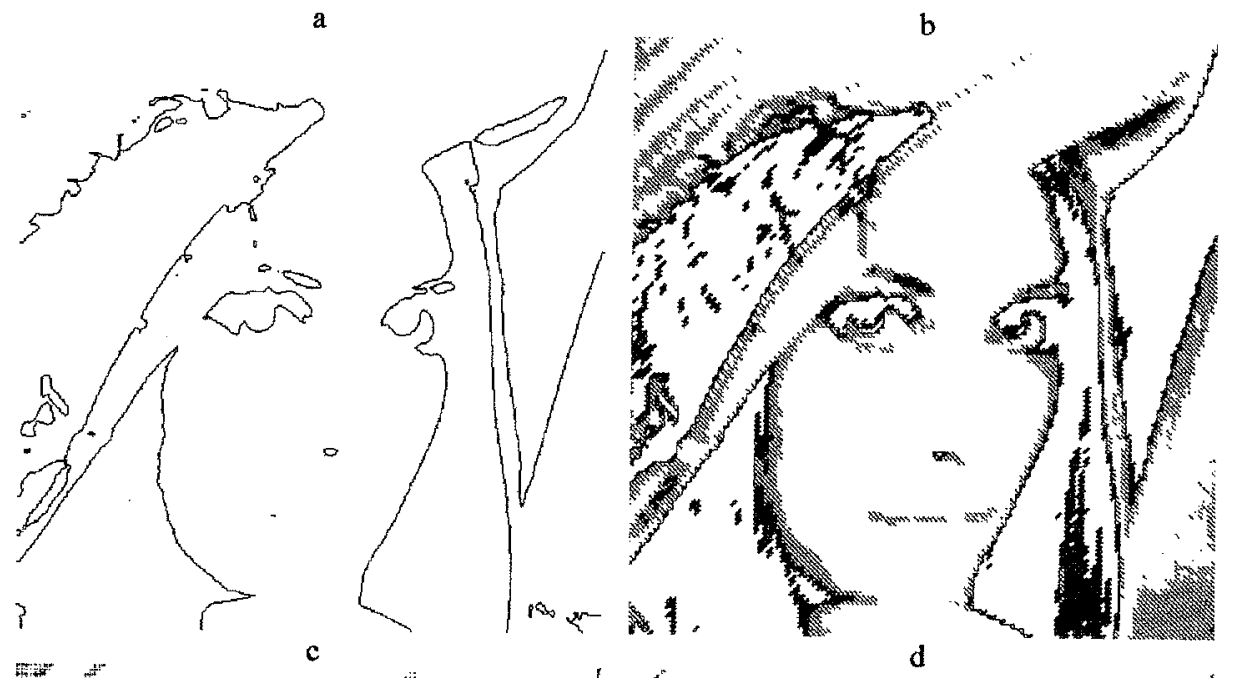

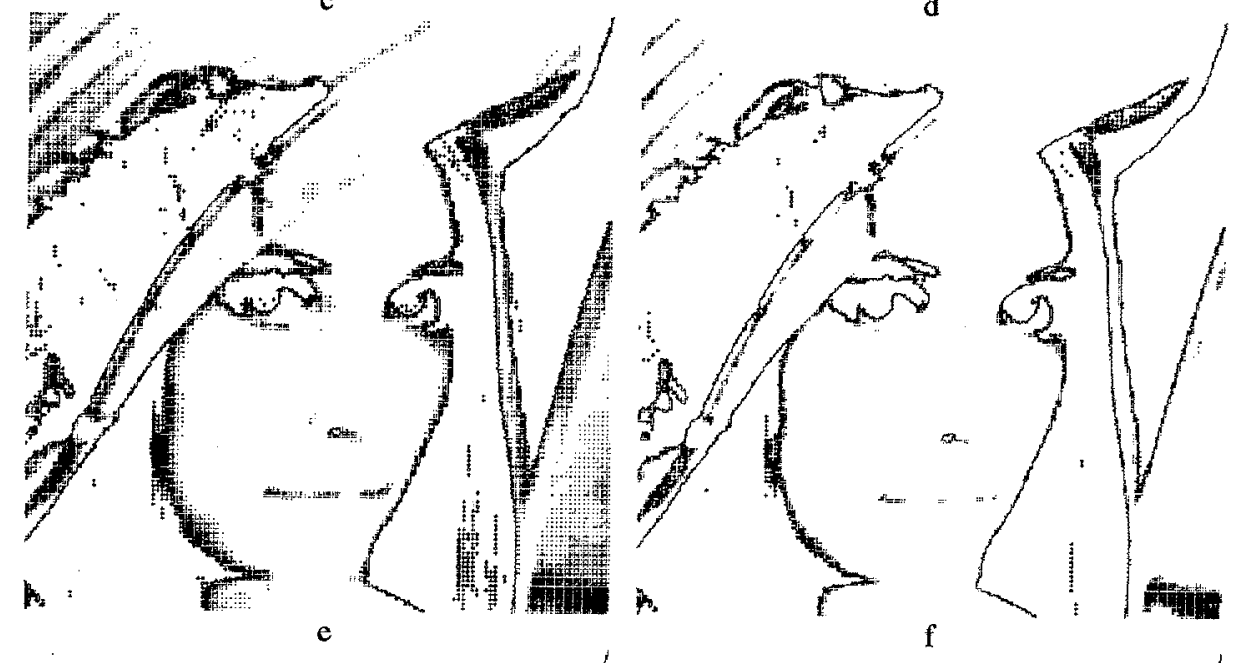

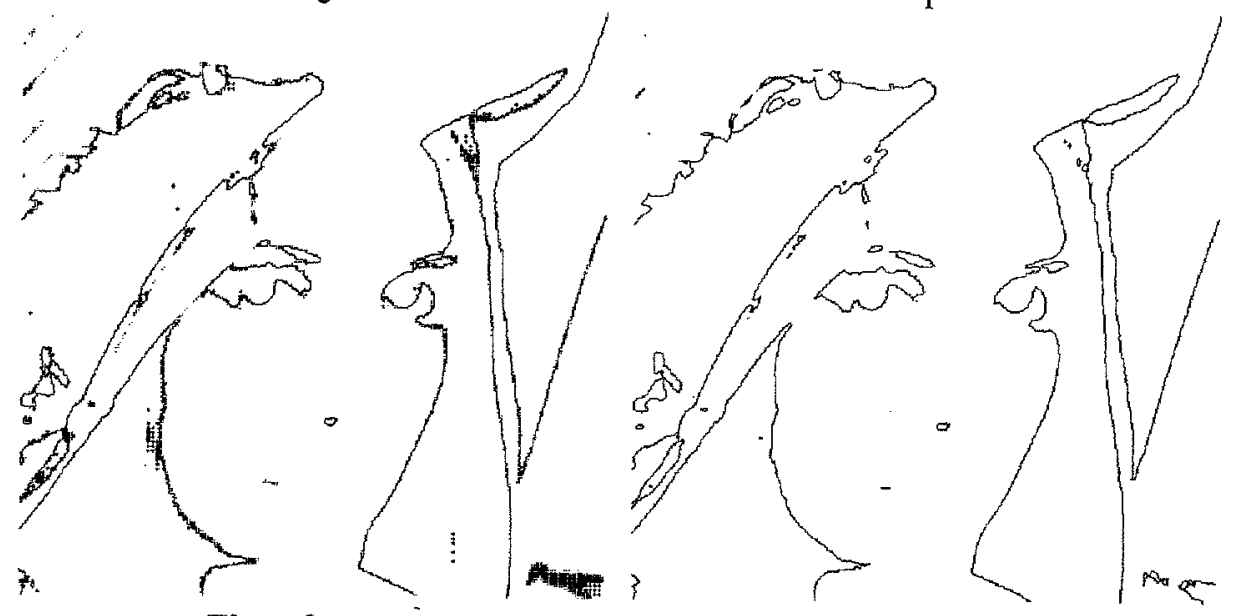

Fig. 3. Edge extractions of the enlargements shown in Fig. 2. 